\title{
Functional roles of multiple Ton complex genes in a Sphingobium degrader of lignin-derived aromatic compounds
}

Masaya Fujita ${ }^{1,4}$, Shodai Yano ${ }^{1}$, Koki Shibata ${ }^{1}$, Mizuki Kondo $^{2}$, Shojiro Hishiyama ${ }^{3}$, Naofumi Kamimura ${ }^{1}$, and Eiji Masai ${ }^{1, *}$

${ }^{1}$ Department of Bioengineering, Nagaoka University of Technology, Nagaoka, Niigata, Japan

${ }^{2}$ Center for Integrated Technology Support, Nagaoka University of Technology, Nagaoka,

Niigata, Japan

${ }^{3}$ Forestry and Forest Products Research Institute, Tsukuba, Ibaraki, Japan

${ }^{4}$ Present address: Structural Biology Research Center, Institute of Materials Structure

Science, High Energy Accelerator Research Organization (KEK), Tsukuba, Ibaraki, Japan

*Corresponding author:

Eiji Masai

Department of Bioengineering, Nagaoka University of Technology, Nagaoka, Niigata

940-2188, Japan

E-mail: emasai@vos.nagaokaut.ac.jp

TEL: +81258479428

ORCID: 0000-0001-9194-7 
TonB-dependent transporters (TBDTs) mediate outer membrane transport of nutrients using the energy derived from proton motive force transmitted from the TonB-ExbB-ExbD complex localized in the inner membrane. Recently, we discovered $d d v T$ encoding a TBDT responsible for the uptake of a 5,5-type lignin-derived dimer in Sphingobium sp. strain

7 SYK-6. Furthermore, overexpression of $d d v T$ in an SYK-6-derivative strain enhanced its uptake capacity, improving the rate of platform chemical production. Thus, understanding the uptake system of lignin-derived aromatics is fundamental for microbial conversion-based lignin valorization. Here we examined whether multiple ton $B$-, exbB-, and exbD-like genes in

11 SYK-6 contribute to the outer membrane transport of lignin-derived aromatics. The

12 disruption of tonB2-6 and exbB3 did not reduce the capacity of SYK-6 to convert or grow on

13 lignin-derived aromatics. In contrast, the introduction of the tonB1-exbB1-exbD1-exbD2

14 operon genes into SYK-6, which could not be disrupted, promoted the conversion of $\beta-\mathrm{O}-4-$,

$15 \beta-5-, \beta-1-, \beta-\beta-$, and 5,5-type dimers and monomers, such as ferulate, vanillate, syringate, and 16 protocatechuate. These results suggest that TonB-dependent uptake involving the tonB1

17 operon genes is responsible for the outer membrane transport of the above aromatics.

18 Additionally, exbB2/tolQ and exbD3/tolR were suggested to constitute the Tol-Pal system that maintains the outer membrane integrity. 
Lignin, a major component of plant cell walls, is the most abundant aromatic compound on Earth and is expected to be a renewable alternative to fossil resources ${ }^{1,2}$. Recently, the production of value-added chemicals from lignin by combining chemical depolymerization of lignin with bacterial catabolic systems for low-molecular-weight aromatic compounds has attracted much attention ${ }^{3}$. Many bacteria catabolizing lignin-derived aromatic compounds have been identified, and their catabolic enzyme genes have been characterized. These genes are useful for the production of value-added chemicals from heterogeneous lignin-derived aromatic compounds ${ }^{4}$. For example, Sphingobium sp. strain SYK-6, an alphaproteobacterium, is a model strain capable of catabolizing various lignin-derived aromatic dimers and monomers and produces 2-pyrone-4,6-dicarboxylic acid (PDC), a platform chemical for the synthesis of functional polymers as an intermediate ${ }^{5,6}$. A significant portion of the SYK-6 genes involved in the catabolism of lignin-derived aromatic compounds have been characterized, and PDC production systems using these genes have been developed ${ }^{4,7}$.

Recently, transporter genes for lignin-derived aromatic compounds have also been studied to improve their uptake capacity ${ }^{8,9}$. However, uptake systems, especially uptake across the outer membrane, remain largely unexplored.

The outer membrane transport by Gram-negative bacteria is mediated by porins, substrate-specific channels, and TonB-dependent transporters (TBDTs) ${ }^{10}$. Porins and substrate-specific channels are responsible for nonspecific and specific passive transport, respectively. TBDTs are active transporters that specifically transport relatively large compounds, such as siderophores and vitamin B12, using the energy derived from proton motive force transmitted from the TonB-ExbB-ExbD complex (Ton complex) localized in the inner membrane ${ }^{11-13}$. Since most bacteria have only a few tonB-like genes compared to the number of TBDT-like genes, one TonB likely interacts with multiple TBDTs to transfer 
the energy required for substrate uptake $\mathrm{e}^{14,15}$. In addition to the above substrates, the involvement of TBDTs in the uptake of oligopeptides, saccharides, and metal ions has been demonstrated, thus, expanding the range of substrates transported by TBDTs ${ }^{16-18}$. It is also known that $t o l A$, tolQ, and $t o l R$, homologs of $\operatorname{ton} B, \operatorname{exb} B$, and $\operatorname{exb} D$, respectively, constitute the Tol-Pal system, stabilizing the outer membrane structure and is involved in the septum formation during cell division ${ }^{19}$. The introduction of $t o l Q$ and $t o l R$ into $e x b B$ and $e x b D$ mutants of Escherichia coli restored the ability of these mutants to take up group B colicin, indicating that $t o l Q$ and $t o l R$ can partially complement the functions of $\operatorname{exb} B$ and $\operatorname{exb} D$, respectively ${ }^{20}$.

The outer membrane transport of aromatic compounds used as carbon and energy sources is mediated by passive transporters, such as the vanillate specific-channel OpdK of Pseudomonas aeruginosa PAO1 and naphthalene porin OmpW of Pseudomonas fluorescens $^{21,22}$. However, SYK-6 has no OpdK-like genes and only one gene that shows similarity with the known aromatic compound porin $(\mathrm{ompW})^{21,23}$, but instead has 74 TBDT-like genes ${ }^{24}$. Recently, we have demonstrated that a TBDT (DdvT) mediates the uptake of 5,5'-dehydrodivanillate (DDVA), a lignin-derived 5,5-type dimer, across the outer membrane in SYK-6. When $d d v T$ was overexpressed in an SYK-6 mutant of the PDC hydrolase gene, the PDC production rate improved. Therefore, overexpression of TBDT genes seems effective in improving the efficiency of microbial conversion. Additionally, the Ton complex composed of TonB1-ExbB1-ExbD1-ExbD2 was suggested to be involved in the uptake of DDVA mediated by DdvT. The phylogenetic analysis of 74 TBDT-like genes in SYK-6 with known TBDT genes revealed that 53 TBDT-like genes form two phylogenetically distinct clades from known TBDT genes. Among them, the expression of 12 TBDT genes in SYK-6 was specifically induced by lignin-derived aromatic compounds.

These facts suggest that the Ton system, consisting of TBDTs and Ton complex, plays a vital role in the uptake of lignin-derived aromatic compounds by SYK-6 across the outer 
membrane. Additionally, the genomes of strains of Sphingomonadaceae, which include many

73 strains capable of degrading recalcitrant aromatic compounds, contain many TBDT-like

74 genes, suggesting that the Ton system is involved in the outer membrane transport of these

75 aromatic compounds ${ }^{25}$. A recent study strongly suggests that TBDTs are involved in the

76 uptake of aromatic compounds, such as benzo[a]pyrene in Novosphingobium

77 pentaromativorans US6-1, which degrades polyaromatic hydrocarbons ${ }^{26}$.

78 This study investigated whether the Ton system is involved in the outer membrane

79 transport of lignin-derived aromatic compounds in SYK-6 by analyzing disruption and

80 overexpression strains of genes presumed to encode components of the Ton complex.

81 Additionally, we predicted the Tol-Pal system genes, which contributed to the stabilization of

82 the outer membrane and characterized their mutants. 
Results and discussion

The ompW-like gene is not required for the conversion of lignin-derived aromatic

compounds.SLG_38320 (omp W) of SYK-6 shows similarity with known aromatic

compound porins (ompW family proteins; 30\% identity with P0A915 of Escherichia coli $)^{21,23,24}$. In our previous study, ompW mutant $(\Delta o m p W)$ exhibited comparable growth to the wild type in Wx medium containing 5 mM DDVA, ferulate (FA), vanillin (VN), vanillate (VA), syringaldehyde (SN), syringate (SA), or protocatechuate (PCA) ${ }^{24}$. Here to clarify whether ompW is involved in the uptake of lignin-derived aromatic compounds, we used the resting cells of $\triangle o m p W$ to measure their capacity to convert lignin-derived 5,5-, $\beta-\mathrm{O}-4-, \beta-\beta-$, $\beta-5-$, and $\beta$-1-type dimers [DDVA, guaiacylglycerol- $\beta$-guaiacyl ether (GGE), pinoresinol (PR), dehydrodiconiferyl alcohol (DCA), and

1,2-bis(4-hydroxy-3-methoxyphenyl)-propane-1,3-diol (HMPPD), respectively] and monomers [acetovanillone (AV), FA, VN, VA, SN, SA, and PCA] (Fig. S1, S2). No reduction in the conversion capacity of $\Delta o m p W$ compared to the wild type was observed, indicating that $\mathrm{omp} W$ is unnecessary to uptake lignin-derived aromatic compounds tested in this study. conversion capacity. SYK-6 has six tonB-like genes (SLG_14430 [tonB1], SLG_34540 [tonB2], SLG_36940 [tonB3], SLG_37490[tonB4], SLG_01650 [tonB5], and SLG_14690 [tonB6]), three exbB-like genes (SLG_14440 [exbB1], SLG_02500 [exbB2/tolQ], and SLG_10800 [exbB3]), and three exbD-like genes (SLG_14450 [exbD1], SLG_14460 [exbD2], and SLG_02490 [exbD3/tolR]) (Table 1, Fig. S3) ${ }^{24}$. Our previous mutant analysis showed that tonB2-6 were not involved in the uptake of $\mathrm{DDVA}^{24}$. tonB1 that constitutes the tonB1 108

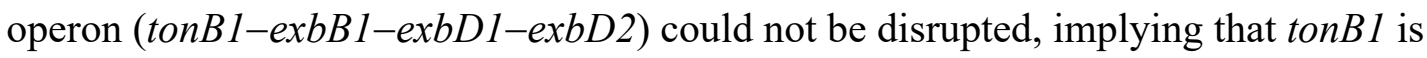


essential for the growth of SYK- $6^{24,27}$. tonB2 and fiuA encoding a TBDT located immediately downstream of tonB2 play a major role in iron acquisition across the outer membrane of SYK- $6^{27}$. Therefore, the growth capacity of tonB2 mutant $(\triangle t o n B 2)$ was reduced on various carbon sources ${ }^{24,27}$.

To examine the involvement of tonBs in the uptake of lignin-derived aromatic compounds, we measured the growth of tonB3 tonB4 tonB5 tonB6 quadruple mutant $(\Delta t o n B 3456)$ and its tonB2 mutant $(\Delta t o n B 23456)$ in $\mathrm{Wx}$ medium containing $5 \mathrm{mM} \mathrm{FA}, \mathrm{VN}$, VA, SA, or PCA, or Wx medium containing SEMP (10 mM sucrose, $10 \mathrm{mM}$ glutamate, 0.13 $\mathrm{mM}$ methionine, and $10 \mathrm{mM}$ proline). We also evaluated the capacity of resting cells of $\Delta$ tonB3456 and $\Delta$ tonB23456 to convert GGE, PR, DCA, HMPPD, AV, FA, VN, VA, SN, SA, and PCA. $\triangle$ tonB3456 and $\triangle$ tonB23456 showed no reduction in growth on all carbon sources compared with wild type and $\triangle t o n B 2$, respectively (Fig. S4). Additionally, the conversion capacity of $\Delta \operatorname{ton} B 3456$ and $\Delta \operatorname{ton} B 23456$ to each compound was not reduced compared to the wild type (Fig. 1). These results suggest that tonB2-6 are not involved in the uptake of these lignin-derived aromatic compounds tested in this study.

The growth of $e x b B$ and $e x b D$ mutants on lignin-derived aromatic compounds. We tried disrupting three $\operatorname{exb} B$-like genes and three $e x b D$-like genes and finally obtained exbB2/tolQ, exbB3, and exbD3/tolR mutants (Table 1, Fig. S5). exbB1, exbD1, and exbD2, which form the ton $B 1$ operon, could not be disrupted, suggesting that this operon plays an essential role in the growth of SYK-6. We measured the growth of exbB2/tolQ mutant ( $\triangle$ exbB2/tolQ), exbB3 mutant $(\triangle e x b B 3)$, and exbD3/tolR mutant $(\triangle e x b D 3 /$ tolR $)$ in $\mathrm{Wx}$ medium containing FA, VN, VA, SA, PCA, or SEMP or diluted LB (Fig. 2). $\triangle \operatorname{exbB3}$ exhibited the same level of growth as the wild type on all substrates. In contrast, the growth of $\Delta \operatorname{exbB2/tolQ}$ and $\Delta \operatorname{exbD3/tolR}$ was significantly reduced or defective in SEMP, diluted LB, and lignin-derived aromatic compounds except for FA. The growth was restored when the plasmids carrying exbB2/tolQ 
and $e x b D 3 / t o l R$ were introduced into $\triangle e x b B 2 /$ tolQ and $\Delta$ exbD3/tolR, respectively (Fig. S6, S7). Therefore, growth retardation or defect was due to the disruption of exbB2/tolQ and exbD3/tolR. However, the introduction of exbB1 into $\triangle \operatorname{exbB2/tolQ}$ and exbD1-exbD2 into $\triangle e x b D 3 /$ tolR did not restore their growth (Fig. S6, S7). These results indicate that exbB1 and exbD1/exbD2 cannot replace the functions of exbB2/tolQ and exbD3/tolR, respectively. exbB2/tolQ and exbD3/tolR were located just upstream of tolA, tolB, and pal-like genes that constitute the Tol-Pal system (Fig. S3), which plays essential roles in the stabilization of the outer membrane structure and septum formation during cell division. Additionary, $y b g C$, whose relevance to the Tol-Pal system is unknown, was found immediately upstream of exbB2/tol ${ }^{19,28}$. Since a similar set of genes $(y b g C-t o l Q-t o l R-t o l A-t o l B-p a l)$ has been found in many bacteria as the genes encoding the Tol-Pal system ${ }^{29}$, exbB2/tolQ and exbD3/tolR are likely components of the Tol-Pal system. Therefore, disruption of these genes is presumed to have caused instability of the outer membrane and associated growth retardation and defects on various carbon sources. The details of the characterization of $\Delta \operatorname{exbB2/tolQ}$ and $\Delta e x b D 3 / t o l R$ will be described later.

In ExbB and TolQ of E. coli, Thr-148 and Thr-181 (ExbB numbering) are essential for proton translocation and Glu-176 (ExbB numbering) whose function is unclear but essential, are conserved ${ }^{30,31}$. These amino acid residues were conserved in ExbB1 and ExbB2/TolQ of SYK-6 (Fig. S8A). However, Glu-176 is not conserved in ExbB3 of SYK-6. With the fact that the growth capacity of $\triangle e x b B 3$ was not reduced on each carbon source compared to the wild type (Fig. 2), likely, ExbB3 does not function as ExbB. In contrast, Asp-25 (ExbD numbering) essential for acquiring proton motive force by ExbD and TolR in E. coli was conserved among ExbD1, ExbD2, and ExbD3/TolR of SYK-6 (Fig. S8B).

\section{The effect of overexpression of the ton $B 1$ operon genes on the conversion of}

lignin-derived aromatic compounds. To examine the involvement of the ton $B 1$ operon 
genes (tonB1-exbB1-exbD1-exbD2) in the uptake of lignin-derived aromatic compounds, each plasmid carrying tonB1 (pJB-tonB1), tonB1-exbB1-exbD1 (pJB-t1-D1), and tonB1-exbB1-exbD1-exbD2 (pJB-t1-D12) was introduced into SYK-6 cells. We measured the capacity of resting cells of SYK-6 harboring pJB-tonB1, pJB-t1-D1, or pJB-t1-D12 to convert DDVA, GGE, PR, DCA, HMPPD, AV, FA, VN, VA, SN, SA, and PCA. The introduction of pJB-tonB1 increased only the conversion rate of DDVA by ca. 1.8 -fold, while the introduction of pJB-t1-D1 or pJB-t1-D12 increased the conversion rate of DDVA, GGE, PR, DCA, HMPPD, FA, VA, SA, and PCA by ca. 1.1-2.6-fold (Table 2, Fig. S9). However, the introduction of these plasmids did not promote the growth of SYK-6 on DDVA, FA, VN, VA, PCA, and SEMP (Fig. S10). On the other hand, the conversion rates of AV, VN, and SN by SYK-6 cells harboring pJB-tonB1, pJB-t1-D1, or pJB-t1-D12 were comparable to those of the control strain. $\mathrm{AV}, \mathrm{VN}$, and $\mathrm{SN}$ are presumed to be taken up by the outer membrane transporters other than TBDT or diffused without transporters.

To determine whether the enhanced conversion capacity of the strains that overexpress the tonB1 operon genes was due to their increased uptake capacity, we assessed the DDVA uptake of these strains using a previously constructed DDVA biosensor ${ }^{9,24}$. Consequently, overexpression of $\operatorname{ton} B 1$, $\operatorname{ton} B 1-e x b B 1-e x b D 1$, and tonB1-exbB1-exbD1-exbD2 increased the DDVA uptake capacity ca. 1.4-, 1.3-, and 1.6-fold, respectively, compared to the control strain (Fig. 3). These results strongly suggest that the tonB1 operon genes are involved in the uptake of DDVA. Similarly, strains overexpressing the tonB1 operon genes likely enhanced the conversion of GGE, PR, DCA, HMPPD, FA, VA, SA, and PCA due to the enhanced capacity to take up each substrate. Therefore, these lignin-derived aromatic compounds seem to be taken up by TBDTs.

We investigated the cellular localization of TonB1. Western blot analysis using anti-TonB1 antibodies against a cell extract and a total membrane fraction obtained from SYK-6 cells grown in LB showed a clear signal in a total membrane fraction, indicating that 
TonB1 is localized in the cell membrane (Fig. S11).

Sphingomonadaceae, to which SYK-6 belongs, contains many strains capable of degrading recalcitrant aromatic compounds such as lignin-derived aromatic compounds and polycyclic aromatic hydrocarbons ${ }^{25}$. These strains have as many or more TBDT and Ton complex genes as SYK-6 $6^{25,27}$. Therefore, we examined whether the tonB1 operon genes of SYK-6 are conserved in the Sphingomonadaceae strains listed in Table S1. These strains have genes showing $43-58 \%$ amino acid sequence identity with $\operatorname{ton} B 1,35-81 \%$ with $\operatorname{exb} B 1$, $35-81 \%$ with exbD1, and 55-75\% with exbD2. Additionally, these genes have a similar gene organization as the SYK-6 tonB1 operon (Fig. S12). Based on these facts, it is highly likely that the tonB1 operon-like genes play a central role in TonB-dependent uptake in these Sphingomonadaceae.

ExbB2/TolQ and ExbD3/TolR constitute the Tol-Pal system. Since mutations in genes constituting the Tol-Pal system have been reported to cause reduced resistance to detergents ${ }^{28}$, we compared the growth of wild type, $\triangle \operatorname{exbB2/tolQ}$, and $\Delta \operatorname{exbD3/tolR}$ in LB with or without $0.3 \mathrm{mg} / \mathrm{ml}$ sodium deoxycholate $(\mathrm{NaDOC})$ or $0.05 \mathrm{mg} / \mathrm{ml}$ sodium dodecyl sulfate (SDS) (Fig. 4). The addition of NaDOC or SDS to the wild type had little effect on growth; however, the growth of $\Delta e x b B 2 /$ tolQ and $\Delta e x b D 3 /$ tolR was significantly reduced in the presence of the detergents.

Mutations in the Tol-Pal system genes cause instability of the outer membrane structure, resulting in the secretion of the outer membrane vesicles (OMVs) $)^{32}$. Therefore, we observed the cell surface structure of the wild type, $\Delta e x b B 2 /$ tolQ, and $\Delta e x b D 3 /$ tolR grown in LB using field emission scanning electron microscopy (FE-SEM) (Fig. 5A). The formation of blebs was observed on the cell surface of $\Delta \operatorname{exbB2/tolQ}$ and $\Delta \operatorname{exbD3/tolR.~To~examine~whether~}$ OMVs were secreted into the culture medium of $\Delta e x b B 2 /$ tolQ and $\Delta e x b D 3 / t o l R$, we performed western blot analysis using anti-DdvT antibodies against the culture supernatants 
of wild type, $\Delta \operatorname{exbB2/tolQ}$, and $\Delta \operatorname{exbD3/tolR}$ grown in LB since OMVs are thought to contain outer membrane proteins (Fig. 6, S13) ${ }^{24,32}$. DdvT was detected in the culture supernatants of $\triangle e x b B 2 /$ tolQ and $\Delta e x b D 3 /$ tolR, but not in the culture supernatant of the wild type. Furthermore, DdvT was not detected in the ultracentrifugated supernatant obtained from the cultures of $\Delta e x b B 2 /$ tolQ and $\Delta e x b D 3 /$ tolR, suggesting the presence of outer membrane fractions in their culture supernatants. Western blot analysis using anti-TonB1 antibodies as an inner membrane marker protein showed that TonB1 was detected only in cell extracts of all strains. These results indicate that OMVs are present in the culture supernatants of $\Delta e x b B 2 /$ tolQ and $\Delta e x b D 3 /$ tolR.

FA was the only carbon source that did not reduce the growth of $\Delta \operatorname{exbB2/tolQ}$ and $\Delta \operatorname{exbD3/tolR~(Fig.~2).~Thus,~vesiculation~of~these~mutants~during~FA~catabolism~was~}$ evaluated. Interestingly, DdvT was not detected in the culture supernatants of $\triangle \operatorname{exbB2/tolQ}$ and $\Delta e x b D 3 /$ tolR grown in LB containing $5 \mathrm{mM} F A$ (Fig. 6). When $\Delta e x b B 2 /$ tolQ and $\triangle e x b D 3 /$ tolR were grown in LB containing $5 \mathrm{mM}$ PCA, a catabolite of FA (Fig. S14A), DdvT was detected in their culture supernatants, suggesting that the outer membrane structure of $\Delta e x b B 2 /$ tolQ and $\Delta e x b D 3 /$ tolR was specifically stabilized during FA catabolism. The observation of the cell surface structure of $\Delta e x b B 2 /$ tolQ and $\Delta e x b D 3 /$ tolR grown in LB containing $5 \mathrm{mM}$ FA showed that the formation of blebs was significantly suppressed during FA catabolism (Fig. 5B). In SYK-6 cells, FA is converted to VN through the addition of coenzyme $\mathrm{A}(\mathrm{CoA})$ to the $\mathrm{C} \gamma$ position and the subsequent release of acetyl-CoA (Fig. S14A) ${ }^{33}$. To get insight into why the growth capacity of $\Delta \operatorname{exbB2/tolQ}$ and $\Delta e x b D 3 /$ tolR was not reduced when FA was used as a carbon source (Fig. 2), we constructed a double mutant of exbD3/tolR and the PDC hydrolase gene ligI ( $\Delta$ exbD3/tolR ligI) (Fig. S5, S14A). This strain produces acetyl-CoA during FA catabolism, but the conversion of VN from FA stops at the PDC. Growth measurement of $\Delta e x b D 3 /$ tolR ligI in the presence or absence of FA showed that the addition of more than $1 \mathrm{mM}$ FA compensated for the growth in diluted LB (Fig. S14B). In 
contrast, the addition of VA and PCA did not positively affect the growth of $\Delta e x b D 3 /$ tolR ligI in diluted LB. Based on these results, the continuous supply of acetyl-CoA produced in the second step of FA catabolism to fatty acid biosynthesis may reduce the damage to the outer membrane caused by the disruption of exbB2/tolQ and exbD3/tolR (Fig. S14A). However, further investigation is needed to elucidate the actual mechanism.

In the Sphingomonadaceae strains that can degrade aromatic compounds listed in Table

$\mathrm{S} 2$, genes with high similarity amino acid sequences (exbB2/tolQ, 56-63\% identity; exbD3/tolR, 46-62\% identity; tolA, 33-52\% identity; tolB, 58-73\% identity; pal, 57-70\% identity) and gene organization with the Tol-Pal system genes of SYK-6 were conserved (Fig. corresponding SYK-6 genes in these bacterial strains.

\section{Conclusions}

Overexpression of the tonB1 operon genes in SYK-6 enhanced the capacity to convert at least five lignin-derived dimers and four monomers. These results strongly suggest that exbB2/tolQ and exbD3/tolR constitute the Tol-Pal system stabilizing the outer membrane structure. Based on this study's results, it is expected that microbial conversion systems with enhanced uptake capacity will be developed through the identification of TBDT genes involved in the outer membrane transport of each lignin-derived aromatic compound. 
Bacterial strains, plasmids, culture conditions, and substrates. The strains and plasmids used in this study are listed in Table S3. Sphingobium sp. SYK-6 (NBRC 103272/JCM $17495)$ and its mutants were grown at $30^{\circ} \mathrm{C}$ with shaking $(160 \mathrm{rpm})$ in $\mathrm{LB}$ or $\mathrm{Wx}$ minimal medium with SEMP ${ }^{34}$. Media for SYK-6 transformants and mutants were supplemented with $50 \mathrm{mg} 1^{-1}$ kanamycin $(\mathrm{Km})$ or $30 \mathrm{mg} \mathrm{l}^{-1}$ chloramphenicol $(\mathrm{Cm})$. E. coli strains were cultured in $\mathrm{LB}$ at $37^{\circ} \mathrm{C}$. Media for $E$. coli transformants carrying antibiotic resistance markers were supplemented with $25 \mathrm{mg} 1^{-1} \mathrm{Km}$ or $30 \mathrm{mg} 1^{-1} \mathrm{Cm}$. Lignin-derived compounds were purchased from Sigma-Aldrich and Tokyo Chemical Industry Co., Ltd. DDVA, PR, DCA, and HMPPD were synthesized as previously described ${ }^{35-38}$.

Mutant construction. Plasmids for gene disruption were constructed by amplifying ca. 1-kb fragments carrying upstream and downstream regions of each gene by PCR with SYK-6 genome DNA as a template and the primer pairs (Table S4). The resultant PCR fragments were inserted into the BamHI site in pAK405 by NEBuilder HiFi DNA assembly cloning kit (New England Biolabs, Inc.). These plasmids were independently introduced into SYK-6 and its mutant cells by triparental mating, and candidate mutants were isolated as previously described ${ }^{39}$. The disruption of the genes was confirmed by colony PCR using primer pairs (Table S4). The plasmids for gene complementation and overexpression (Table S3) were introduced into SYK-6 and the mutant cells by electroporation.

Sequence analysis. DNA sequencing of PCR amplification products was conducted by Additionally, sequence similarity searches, multiple alignments, and pairwise alignments were conducted using the BLAST ${ }^{40}$, Clustal Omega ${ }^{41}$, and EMBOSS programs ${ }^{42}$, 
respectively.

Growth measurement. Cells of SYK-6, its mutants, and complemented strains were grown in LB for $24 \mathrm{~h}$. The cells were harvested by centrifugation at $4,800 \times g$ for $5 \mathrm{~min}$, washed twice with Wx buffer, and resuspended in $3 \mathrm{ml}$ of the same buffer. The cells were then inoculated into diluted LB, Wx medium containing SEMP, or Wx medium containing $5 \mathrm{mM}$ DDVA, FA, VN, VA, SA, or PCA to an $\mathrm{OD}_{660}$ of 0.2. Since SYK-6 exhibits auxotrophy for methionine when grown in a methoxy-group-free substrate, $0.13 \mathrm{mM}$ methionine was added to the medium to grow on PCA. Cells were incubated at $30{ }^{\circ} \mathrm{C}$ with shaking $(60 \mathrm{rpm})$, and cell growth was monitored every hour by measuring the OD 660 with a TVS062CA biophotorecorder (Advantec Co., Ltd.). For the assay of complemented strains, Km and 1 $\mathrm{mM} m$-toluate (an inducer of the $\mathrm{P}_{m}$ promoter in $\mathrm{pJB} 861$ ) were added to the medium; for the assay of SYK-6 cells overexpressing the tonB 1 operon genes, $\mathrm{Km}$ and $0.5 \mathrm{mM} m$-toluate were added to the medium.

Resting cell assay. Cells of SYK-6 and its mutant were grown in LB for $20 \mathrm{~h}$ and harvested by centrifugation at $14,100 \times g$ for 5 min. The cells were washed twice with $50 \mathrm{mM}$ Tris- $\mathrm{HCl}$ buffer ( $\mathrm{pH} 7.5)$ and resuspended in $1 \mathrm{ml}$ of the same buffer. For conversion of AV, cells were grown in Wx-SEMP until $\mathrm{OD}_{600}$ reached 0.5 and then incubated in the presence of $5 \mathrm{mM} \mathrm{AV}$ for $12 \mathrm{~h}$. The cells were then incubated in $50 \mathrm{mM}$ Tris-HCl buffer (pH 7.5) with $100 \mu \mathrm{M}$ DDVA (OD 600 of the cells, 5.0; reaction time, 6 h), $200 \mu \mathrm{M}$ GGE (5.0; $6 \mathrm{~h}), 100 \mu \mathrm{M}$ PR (0.5; 3 h), $100 \mu \mathrm{M}$ DCA (0.5; 2 h), $100 \mu \mathrm{M} \operatorname{HMPPD}(2.0 ; 2$ h), $100 \mu \mathrm{M}$ AV (0.5; 3 h) , $100 \mu \mathrm{M}$ FA (1.0; 2 h), $200 \mu \mathrm{M} \mathrm{VN} \mathrm{(0.2;} 1$ h), $100 \mu \mathrm{M}$ VA (2.0: 2 h), $200 \mu \mathrm{M} \mathrm{SN}(0.4 ; 1$ h), $100 \mu \mathrm{M} \mathrm{SA}$ $(2.0 ; 2 \mathrm{~h})$, or $100 \mu \mathrm{M}$ PCA $(2.0 ; 1 \mathrm{~h})$. Samples were collected periodically, and the reactions were stopped by centrifugation at $18,800 \times g$ for $10 \mathrm{~min}$. The supernatants were diluted 5-fold in water, filtered, and analyzed using high-performance liquid chromatography 
(HPLC). For the analysis of SYK-6 cells harboring plasmids carrying the tonB1 operon genes, the cells grown in LB containing $\mathrm{Km}$ and $0.5 \mathrm{mM} m$-toluate were employed. These cells were incubated with lignin-derived aromatic compounds under the conditions described above, with the following exceptions. For the conversion of $100 \mu \mathrm{M}$ DDVA, $100 \mu \mathrm{M}$ GGE, $100 \mu \mathrm{M}$ $\mathrm{PR}$, and $100 \mu \mathrm{M}$ PCA, cells with $\mathrm{OD}_{600}$ of 5.0, 3.0, 0.2, and 1.0, respectively, were used and incubated with each substrate for 5, 3, 4, and $2 \mathrm{~h}$.

HPLC conditions. HPLC analysis was conducted using an Acquity UPLC system (Waters Corporation) with a TSKgel ODS-140HTP column (2.1 by $100 \mathrm{~mm}$; Tosoh Corporation). All

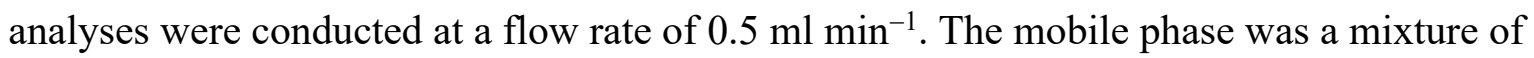
solution A (acetonitrile containing $0.1 \%$ formic acid) and solution B (water containing $0.1 \%$ formic acid) under the following conditions: i) conversion of DDVA, 0-2.5 min, 15\% A; ii) conversion of GGE, 0-3.2 min, linear gradient from 5 to $40 \% \mathrm{~A} ; 3.2-6.0 \mathrm{~min}$, decreasing gradient from 40 to $5 \% \mathrm{~A} ; 6.0-7.0 \mathrm{~min}, 5 \% \mathrm{~A}$. iii) conversion of PR, 0-5.0 min, 20\%A. iv) conversion of DCA, 0-3.0 min, 25\% A. v) conversion of HMPPD, 0-2.5 min, 10\% A. vi) conversion of AV, $0-2.0 \mathrm{~min}, 15 \% \mathrm{~A}$. vii) conversion of FA, $0-2.5 \mathrm{~min}, 15 \% \mathrm{~A}$. viii) conversion of $\mathrm{VN}$ and $\mathrm{SN}, 0-2.0 \mathrm{~min}, 15 \% \mathrm{~A}$. ix) conversion of VA and SA, $0-2.0 \mathrm{~min}, 10 \%$ A. $\mathrm{x}$ ) conversion of PCA, 0-1.5 min, 10\% A. DDVA, GGE, PR, DCA, HMPPD, AV, FA, VN, VA, SN, SA, and PCA were detected at 265, 270, 280, 277, 278, 276, 322, 280, 260, 308, 275, and $260 \mathrm{~nm}$, respectively.

DDVA uptake assay. Cells of SYK-6 harboring pS-XR and pS-tonB1, pS-t1-D1, or pS-t1-D12 grown in LB containing $\mathrm{Km}$ and $\mathrm{Cm}$ for $20 \mathrm{~h}$ were harvested by centrifugation at $4,800 \times g$ for $5 \mathrm{~min}$, washed twice with $\mathrm{Wx}$ buffer, and resuspended in $1 \mathrm{ml} \mathrm{Wx-SEMP.} \mathrm{The}$ cells were then inoculated into Wx-SEMP containing $0.5 \mathrm{mM} m$-toluate with or without 100 $\mu \mathrm{M}$ DDVA to an $\mathrm{OD}_{600}$ of 2.0. Samples were incubated at $30^{\circ} \mathrm{C}$ with shaking $(1,500 \mathrm{rpm})$ 
for $3 \mathrm{~h}$. The $\beta$-galactosidase activity of the cells was measured using

2-nitrophenyl- $\beta$-D-galactopyranoside as the substrate, according to a modified Miller assay

[https://openwetware.org/wiki/Beta-Galactosidase_Assay_(A_better_Miller)] ${ }^{9}$.

$\beta$-galactosidase activity was expressed as Miller units.

Detergent resistance assay. Cells of SYK-6 and its mutants were grown in LB for $20 \mathrm{~h}$ were harvested by centrifugation at $4,800 \times g$ for 5 min and resuspended in Wx buffer. The cells were inoculated into LB with or without $0.05 \mathrm{mg} \mathrm{ml}^{-1} \mathrm{SDS}$ or $0.3 \mathrm{mg} \mathrm{ml}^{-1} \mathrm{NaDOC}$ to an $\mathrm{OD}_{600}$ of 0.2 and incubated at $30{ }^{\circ} \mathrm{C}$ for $24 \mathrm{~h}$. The cultured cells were then serially diluted with Wx buffer, and $10 \mu 1$ of each cell suspension was dropped onto an LB plate. The plates were incubated at $30^{\circ} \mathrm{C}$ for $72 \mathrm{~h}$.

Western blot analysis. A peptide corresponding to residues 199-210 (QAGNPIRTKDRR) of

TonB1 was synthesized and used as an antigen to obtain antisera for TonB1 in rabbits (Cosmo Bio, Inc.). Anti-TonB1-peptide antibodies were obtained by purifying the antiserum using peptide affinity column chromatography (Cosmo Bio, Inc.). Anti-DdvT antibodies were obtained in a previous study ${ }^{24}$. Total membrane fractions were prepared as described previously from SYK-6 cells incubated in LB for $20 \mathrm{~h}^{24}$. TonB1 and DdvT were detected by western blot analysis using anti-TonB1 antibodies $\left(0.11 \mu \mathrm{g} \mathrm{ml}^{-1}\right)$ and anti-DdvT antibodies $\left(0.25 \mu \mathrm{g} \mathrm{ml}^{-1}\right)$ as described previously ${ }^{24}$. Horseradish peroxidase-conjugated goat anti-rabbit IgG antibodies (Invitrogen, $0.2 \mu \mathrm{g} \mathrm{ml}^{-1}$ ) were used as the secondary antibodies. Protein concentrations were determined by the Bradford method using a Bio-Rad protein assay kit or Lowry's assay with a DC protein assay kit (Bio-Rad Laboratories). TonB1 and DdvT were detected using the ECL Western Blotting Detection System (GE Healthcare) with a LumiVision PRO image analyzer (Aisin Seiki Co., Ltd). 
Detection of outer membrane vesiculation of $\triangle e x b B 2 / t o l Q$ and $\triangle e x b D 3 / t o l R$. Cells of SYK-6 and its mutants were grown in LB with or without $5 \mathrm{mM} \mathrm{FA}$ or PCA for $20 \mathrm{~h}$ were harvested by centrifugation at $19,000 \times g$ for $10 \mathrm{~min}$. The supernatant of the centrifugated culture was filtered with a $0.45 \mu \mathrm{m}$ membrane filter (supernatant fraction). Then, the supernatant fraction was ultracentrifuged at $120,000 \times g$ for 60 min to obtain ultracentrifuged supernatant (ultrasupernatant fraction). The cells were washed twice with $50 \mathrm{mM}$ Tris-HCl buffer ( $\mathrm{pH} 7.5$ ) containing $150 \mathrm{mM} \mathrm{NaCl}$ and resuspended in the same buffer containing 1 mM phenylmethylsulfonyl fluoride. The cells were disrupted by sonication, and cell lysate was obtained. Each fraction was separated by SDS-7.5\% or $15 \%$ polyacrylamide gel electrophoresis and performed western blot analysis using anti-TonB1 antibodies and anti-DdvT antibodies as described above.

FE-SEM analysis. Cells of SYK-6 and its mutants grown on LB plates with or without 5 mM FA for $48 \mathrm{~h}$ were deposited on poly-D-lysine coated glass (Cosmo Bio, Inc.) and fixed with $2.0 \%$ glutaraldehyde solution in $200 \mathrm{mM}$ sodium phosphate buffer $(\mathrm{pH} 7.5)$ for $2 \mathrm{~h}$ at room temperature. Fixed cells were washed twice with the same buffer and dehydrated in an ethanol gradient of $25 \%, 50 \%, 75 \%, 90 \%$, and $100 \%$ for 15 min per step. Samples were dried using an evaporator for $8 \mathrm{~h}$ at $30{ }^{\circ} \mathrm{C}$ and sputter-coated with Os. Cell images were obtained using a HITACHI SU8230 field emission SEM with a beam accelerating voltage of $1.0 \mathrm{kV}$.

\section{Statistics and reproducibility}

All results were obtained from $n=3$ independent experiments. Statistical tests were performed using GraphPad Prism9 (GraphPad software). Unpaired, two-tailed $t$-test were used as shown in figure legends. $P<0.05$ was considered statistically significant.

\section{Data availability}


391 All data supporting this study are available within the article and its Supplementary

392 Information or are available from the corresponding author upon request.

393 


\section{References}

395

3961 Vanholme, R., Demedts, B., Morreel, K., Ralph, J. \& Boerjan, W. Lignin biosynthesis and structure. Plant Physiol. 153, 895-905, doi:10.1104/pp.110.155119 (2010).

3982 Calvo-Flores, F. G. \& Dobado, J. A. Lignin as renewable raw material. ChemSusChem 3, 1227-1235, doi:10.1002/cssc.201000157 (2010).

4003 Beckham, G. T., Johnson, C. W., Karp, E. M., Salvachúa, D. \& Vardon, D. R. Opportunities and challenges in biological lignin valorization. Curr. Opin. Biotechnol. 42, 40-53, doi:10.1016/j.copbio.2016.02.030 (2016).

403 Kamimura, N. et al. Bacterial catabolism of lignin-derived aromatics: New findings in a recent decade: Update on bacterial lignin catabolism. Environ. Microbiol. Rep. 9, 679-705, doi:10.1111/1758-2229.12597 (2017).

406 Masai, E., Katayama, Y. \& Fukuda, M. Genetic and biochemical investigations on bacterial catabolic pathways for lignin-derived aromatic compounds. Biosci. Biotechnol. Biochem. 71, 1-15 (2007).

Otsuka, Y. et al. Efficient production of 2-pyrone 4,6-dicarboxylic acid as a novel polymer-based material from protocatechuate by microbial function. Appl. Microbiol. Biotechnol. 71, 608-614, doi:10.1007/s00253-005-0203-7 (2006).

Higuchi, Y. et al. Discovery of novel enzyme genes involved in the conversion of an arylglycerol- $\beta$-ether metabolite and their use in generating a metabolic pathway for lignin valorization. Metab. Eng. 55, 258-267, doi:10.1016/j.ymben.2019.08.002 (2019).

Mori, K., Kamimura, N. \& Masai, E. Identification of the protocatechuate transporter gene in Sphingobium sp. strain SYK-6 and effects of overexpression on production of a value-added metabolite. Appl. Microbiol. Biotechnol. 102, 4807-4816, doi:10.1007/s00253-018-8988-3 (2018). Mori, K., Niinuma, K., Fujita, M., Kamimura, N. \& Masai, E. DdvK, a novel major facilitator superfamily transporter essential for 5,5'-dehydrodivanillate uptake by Sphingobium sp. strain SYK-6. Appl. Environ. Microbiol. 84, doi:10.1128/AEM.01314-18 (2018).

Nikaido, H. Molecular basis of bacterial outer membrane permeability revisited. Microbiol. Mol. Biol. Rev. 67, 593-656, doi:10.1128/mmbr.67.4.593-656.2003 (2003). signalling domain provides insights into TonB-mediated siderophore uptake. eLife 8, e48528, doi:10.7554/eLife.48528 (2019).

Shultis, D. D., Purdy, M. D., Banchs, C. N. \& Wiener, M. C. Outer membrane active transport: structure of the BtuB:TonB complex. Science 312, 1396-1399, doi:10.1126/science.1127694 (2006).

Ratliff, A. C., Buchanan, S. K. \& Celia, H. Ton motor complexes. Curr. Opin. Struct. Biol. 67, 95-100, doi:10.1016/j.sbi.2020.09.014 (2021).

Chu, B. C., Peacock, R. S. \& Vogel, H. J. Bioinformatic analysis of the TonB protein family. Biometals 20, 467-483, doi:10.1007/s10534-006-9049-4 (2007).

5 Blanvillain, S. et al. Plant carbohydrate scavenging through TonB-dependent receptors: a feature 
shared by phytopathogenic and aquatic bacteria. PLoS One 2, e224, doi:10.1371/journal.pone.0000224 (2007).

6 Bolam, D. N. \& van den Berg, B. TonB-dependent transport by the gut microbiota: novel aspects of an

17 Calmettes, C. et al. The molecular mechanism of zinc acquisition by the neisserial outer-membrane transporter ZnuD. Nat. Commun. 6, 7996, doi:10.1038/ncomms8996 (2015).

18 Madej, M. et al. Structural and functional insights into oligopeptide acquisition by the RagAB transporter from Porphyromonas gingivalis. Nat. Microbiol. 5, 1016-1025, doi:10.1038/s41564-020-0716-y (2020).

19 Szczepaniak, J., Press, C. \& Kleanthous, C. The multifarious roles of Tol-Pal in Gram-negative bacteria.

20 Braun, V. \& Herrmann, C. Evolutionary relationship of uptake systems for biopolymers in Escherichia coli: cross-complementation between the TonB-ExbB-ExbD and the TolA-TolQ-TolR proteins. Mol. Microbiol. 8, 261-268, doi:10.1111/j.1365-2958.1993.tb01570.x (1993).

21 Neher, T. M. \& Lueking, D. R. Pseudomonas fluorescens ompW: plasmid localization and requirement for naphthalene uptake. Can. J. Microbiol. 55, 553-563, doi:10.1139/w09-002 (2009).

22 Tamber, S., Ochs, M. M. \& Hancock, R. E. Role of the novel OprD family of porins in nutrient uptake in Pseudomonas aeruginosa. J. Bacteriol. 188, 45-54, doi:10.1128/JB.188.1.45-54.2006 (2006).

23 Hong, H., Patel, D. R., Tamm, L. K. \& van den Berg, B. The outer membrane orotein OmpW forms an eight-stranded $\beta$-barrel with a hydrophobic channel. J. Biol. Chem. 281, 7568-7577, doi:10.1074/jbc.M512365200 (2006).

24 Fujita, M. et al. A TonB-dependent receptor constitutes the outer membrane transport system for a lignin-derived aromatic compound. Commun. Biol. 2, 432, doi:10.1038/s42003-019-0676-z (2019).

25 Samantarrai, D., Lakshman Sagar, A., Gudla, R. \& Siddavattam, D. TonB-dependent transporters in Sphingomonads: unraveling their distribution and function in environmental adaptation. Microorganisms 8, doi:10.3390/microorganisms8030359 (2020).

26 Liang, J. et al. Benzo[a]pyrene might be transported by a TonB-dependent transporter in Novosphingobium pentaromativorans US6-1. J. Hazard. Mater. 404, 124037, doi:10.1016/j.jhazmat.2020.124037 (2021).

27 Fujita, M. et al. Iron acquisition system of Sphingobium sp. strain SYK-6, a degrader of lignin-derived aromatic compounds. Sci. Rep. 10, 12177, doi:10.1038/s41598-020-68984-2 (2020).

8 Szczepaniak, J. et al. The lipoprotein Pal stabilises the bacterial outer membrane during constriction by a mobilisation-and-capture mechanism. Nat. Commun. 11, 1305, doi:10.1038/s41467-020-15083-5 (2020).

29 Gao, T., Meng, Q. \& Gao, H. Thioesterase YbgC affects motility by modulating c-di-GMP levels in Shewanella oneidensis. Sci. Rep. 7, 3932, doi:10.1038/s41598-017-04285-5 (2017).

30 Baker, K. R. \& Postle, K. Mutations in Escherichia coli ExbB transmembrane domains identify scaffolding and signal transduction functions and exclude participation in a proton pathway. $J$. 
Bacteriol. 195, 2898-2911, doi:10.1128/jb.00017-13 (2013).

Celia, H. et al. Structural insight into the role of the Ton complex in energy transduction. Nature 538, 60-65, doi:10.1038/nature19757 (2016).

32 Bernadac, A., Gavioli, M., Lazzaroni, J.-C., Raina, S. \& Lloubès, R. Escherichia coli tol-pal mutants form outer membrane vesicles. J. Bacteriol 180, 4872-4878, doi:10.1128/JB.180.18.4872-4878.1998 (1998).

33 Masai, E. et al. Cloning and characterization of the ferulic acid catabolic genes of Sphingomonas paucimobilis SYK-6. Appl. Environ. Microbiol. 68, 4416-4424, doi:10.1128/aem.68.9.4416-4424.2002 (2002).

34 Kasai, D. et al. Characterization of FerC, a MarR-type transcriptional regulator, involved in transcriptional regulation of the ferulate catabolic operon in Sphingobium sp. strain SYK-6. FEMS Microbiol. Lett. 332, 68-75, doi:10.1111/j.1574-6968.2012.02576.x (2012).

35 Peng, X. et al. Cloning of a Sphingomonas paucimobilis SYK-6 gene encoding a novel oxygenase that cleaves lignin-related biphenyl and characterization of the enzyme. Appl. Environ. Microbiol. 64, 2520-2527 (1998).

6 Fukuhara, Y. et al. Discovery of pinoresinol reductase genes in Sphingomonads. Enzyme Microb. Technol. 52, 38-43, doi:10.1016/j.enzmictec.2012.10.004 (2013).

37 Takahashi, K. et al. Characterization of the catabolic pathway for a phenylcoumaran-type lignin-derived biaryl in Sphingobium sp. strain SYK-6. Biodegradation 25, 735-745, doi:10.1007/s10532-014-9695-0 (2014).

Nakatsubo, F. \& Higuchi, T. Synthesis of 1,2-diarylpropane-1,3-diols and determination of their configurations. Holzforschung 29, 193-198, doi:10.1515/hfsg.1975.29.6.193 (1975).

Kaczmarczyk, A., Vorholt, J. A. \& Francez-Charlot, A. Markerless gene deletion system for Sphingomonads. Appl. Environ. Microbiol. 78, 3774-3777, doi:10.1128/AEM.07347-11 (2012).

Johnson, M. et al. NCBI BLAST: a better web interface. Nucleic Acids Res. 36, W5-9, doi:10.1093/nar/gkn201 (2008).

41 Sievers, F. et al. Fast, scalable generation of high-quality protein multiple sequence alignments using Clustal Omega. Mol. Syst. Biol. 7, 539, doi:10.1038/msb.2011.75 (2011).

42 Li, W. et al. The EMBL-EBI bioinformatics web and programmatic tools framework. Nucleic Acids Res. 43, W580-584, doi:10.1093/nar/gkv279 (2015). 


\section{Acknowledgments}

502 This work was supported by JSPS KAKENHI Grant Numbers 15H04473 (E.M.), 19H02867

503 (E.M.), 19J11312 (M.F.), and 21J00894 (M.F.). Part of this work resulted from using research

504 equipment shared in MEXT Project for promoting public utilization of advanced research

505 infrastructure (Program for the SHARE, GIGAKU-Innovation Equipment Sharing Network)

506 at Nagaoka University of Technology, Grant Number [JPMXS0430300121].

508 Author contributions

509 E.M. supervised the project. M.F., N.K., and E.M. designed the study and wrote the

510 manuscript. M.F. performed the experiments with the following exceptions. S.Y. and K.S.

511 analyzed PR, DCA, and AV conversion by tonB mutants and overexpression strains of the

512 tonB1 operon genes. M.K. and M.F. performed FE-SEM analysis. S.H. synthesized DCA, PR,

513 and HMPPD. S.Y. performed a statistical analysis of the conversion of lignin-derived

514 aromatic compounds by overexpression strains of the tonB1 operon genes. S.Y., K.S., and

515 K.M. helped to interpret the data and discussed the results. All authors read and approved the

516 manuscript.

\section{Competing interests}

519 The authors declare no competing interests. 
bioRxiv preprint doi: https://doi.org/10.1101/2021.08.06.455174; this version posted August 6,2021 . The copyright holder for this preprint (which was not certified by peer review) is the author/funder. All rights reserved. No reuse allowed without permission.

Table 1. Candidate genes encoding the Ton complex components in Sphingobium sp. strain SYK-6

\begin{tabular}{|c|c|c|c|c|c|c|}
\hline Locus tag & $\begin{array}{l}\text { Putative } \\
\text { function }\end{array}$ & $\begin{array}{c}\text { Most } \\
\text { similar } \\
\text { protein }^{a}\end{array}$ & Annotation & Strain & $\begin{array}{c}\text { Sequence } \\
\text { identity } \\
(\%)\end{array}$ & E-value \\
\hline \multicolumn{7}{|l|}{ TonB } \\
\hline SLG_01650 (tonB5) & TonB & - & - & - & - & - \\
\hline SLG_14430 (tonB 1$)$ & TonB & - & - & - & - & - \\
\hline SLG_14690 (tonB6) & TonB & - & - & - & - & - \\
\hline SLG_34540(tonB2) & TonB & - & - & - & - & - \\
\hline SLG_36940 (tonB3) & TonB & - & - & - & - & - \\
\hline SLG_37490(tonB4) & TonB & - & - & - & - & - \\
\hline \multicolumn{7}{|l|}{ ExbB } \\
\hline SLG_02500 (exbB2/tolQ) & ExbB/TolQ & P50598 & Tol-Pal system protein TolQ & Pseudomonas aeruginosa $\mathrm{PAO} 1$ & 41 & $4 \mathrm{E}-46$ \\
\hline SLG_10800 (exbB3) & ExbB & - & - & - & - & - \\
\hline SLG_14440 (exbB1) & ExbB & P0C7L9 & Biopolymer transport protein ExbB & $\begin{array}{l}\text { Xanthomonas campestris pv. } \\
\text { campestris str. ATCC } 33913\end{array}$ & 38 & $4 \mathrm{E}-42$ \\
\hline \multicolumn{7}{|l|}{ ExbD } \\
\hline SLG_02490 (exbD3/tolR $)$ & ExbD/TolR & P0ABV2 & Biopolymer transport protein ExbD & Escherichia coli $\mathrm{K}-12$ & 41 & $7 \mathrm{E}-27$ \\
\hline SLG_14450 (exbD1) & ExbD & P0C7I9 & Biopolymer transport protein ExbD2 & $\begin{array}{l}\text { Xanthomonas campestris pv. } \\
\text { campestris str. ATCC } 33913\end{array}$ & 29 & $6 \mathrm{E}-17$ \\
\hline SLG_14460 (exbD2) & ExbD & P0C7M0 & Biopolymer transport protein ExbD1 & $\begin{array}{l}\text { Xanthomonas campestris pv. } \\
\text { campestris str. ATCC } 33913\end{array}$ & 37 & $3 \mathrm{E}-24$ \\
\hline
\end{tabular}

${ }^{a}$ Most similar proteins were searched in the Swiss-Prot database using the BLAST-P program ${ }^{40}$ and not displayed if the E-value is greater than $1 \mathrm{e}-10$. 
Table 2. The conversion rates of lignin-derived aromatic compounds by $S Y K-6$ cells overexpressing the tonB1 operon genes

\begin{tabular}{lcccc}
\hline \multicolumn{1}{c}{ Substrate } & WT + pJB861 & WT + pJB-tonB1 & WT + pJB-t1-D1 & WT + pJB-t1-D12 \\
\hline DDVA & $7.0 \pm 1.1$ & $12 \pm 0.6^{* *}[1.8]$ & $15 \pm 0.1 * * *[2.2]$ & $18 \pm 1.4 * * *[2.6]$ \\
GGE & $45 \pm 2.4$ & $49 \pm 1.7$ & $53 \pm 1.1 *[1.2]$ & $53 \pm 0.2 * *[1.2]$ \\
PR & $35 \pm 1.2$ & $36 \pm 0.8$ & $43 \pm 0.7 * *[1.2]$ & $43 \pm 2.2^{*}[1.2]$ \\
DCA & $133 \pm 3.4$ & $142 \pm 7.4$ & $164 \pm 1.8 * * *[1.2]$ & $161 \pm 0.5 * * *[1.2]$ \\
HMPPD & $98 \pm 4.6$ & $101 \pm 5.8$ & $81 \pm 13$ & $111 \pm 4.2^{*}[1.1]$ \\
AV & $42 \pm 4.4$ & $41 \pm 1.1$ & $42 \pm 2.6$ & $41 \pm 1.0$ \\
FA & $73 \pm 2.3$ & $74 \pm 1.3$ & $74 \pm 2.8$ & $86 \pm 1.0^{* *}[1.2]$ \\
VN & $229 \pm 13$ & $257 \pm 15$ & $187 \pm 35$ & $229 \pm 8.0$ \\
VA & $38 \pm 2.9$ & $38 \pm 1.9$ & $212 \pm 16$ & $47 \pm 0.5 *[1.2]$ \\
SN & $210 \pm 16$ & $197 \pm 20$ & $51 \pm 1.7$ & $193 \pm 8.6$ \\
SA & $44 \pm 4.0$ & $44 \pm 2.0$ & $75 \pm 1.3$ & $59 \pm 1.8^{* *}[1.4]$ \\
PCA & $73 \pm 1.8$ & $72 \pm 2.9$ & $85 \pm 2.2^{* *[1.2]}$ \\
\hline The conv
\end{tabular}

The conversion rates were calculated from the results of Fig. S9 using the decreasing amount of each substrate at the time interval (DDVA, 0-5.0 h; GGE, 0-1.5 h; PR, 0-2.0 h; DCA, 0-0.5 h; HMPPD, 0-0.5 h; AV, 0-2.0 h; FA, 0-1.0 h; VN, 0-0.5 h; VA, 0-2.0 h; SN, 0-0.5 h; SA, 0-1.5 h; PCA, 0-1.0 h), where almost linear conversions of the substrates were observed. Each value is the average \pm the standard deviation of three independent experiments. ${ }^{*} P<0.05$, $* * P<$ $0.01, * * * P<0.001$ (values indicating significance between the conversion rates of SYK-6 cells harboring pJB861 and SYK-6 cells harboring pJB-tonB1, pJB-t1-D1, or pJB-t1-D12 from unpaired, two-tailed $t$-test). The $P$ value of each field without an asterisk, $P>0.05$. Values in the brackets indicate fold change between the conversion rates by SYK-6 cells harboring pJB861 and SYK-6 cells harboring pJB-tonB1, pJB-t1-D1, or pJB-t1-D12. 

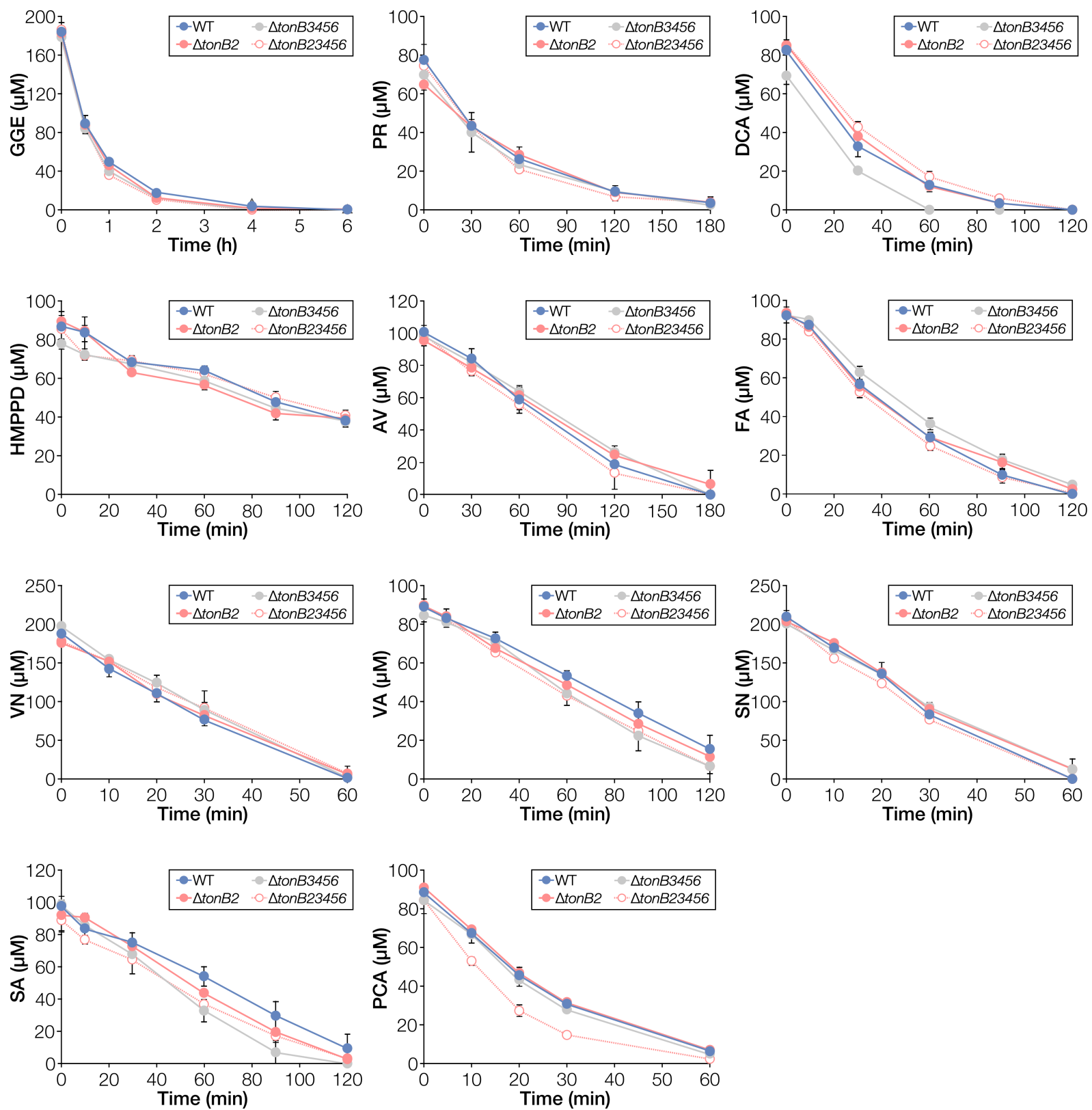

521 Fig. 1 Conversion of lignin-derived aromatic compounds by resting cells of tonB multiple

522 mutants. Cells of SYK-6, $\Delta \operatorname{tonB2}, \Delta \operatorname{tonB} 3456$, and $\Delta$ tonB23456 were incubated with $200 \mu \mathrm{M}$ GGE, $523100 \mu \mathrm{M}$ PR, $100 \mu \mathrm{M}$ DCA, $100 \mu \mathrm{M}$ HMPPD, $100 \mu \mathrm{M}$ AV, $100 \mu \mathrm{M}$ FA, $200 \mu \mathrm{M}$ VN, $100 \mu \mathrm{M}$ VA, $524200 \mu \mathrm{M}$ SN, $100 \mu \mathrm{M}$ SA, or $100 \mu \mathrm{M}$ PCA. Portions of the reaction mixtures were collected, and the 525 amount of substrate was measured using HPLC. Each value is the average \pm the standard deviation of 526 three independent experiments. GGE, guaiacylglycerol- $\beta$-guaiacyl ether; PR, pinoresinol; DCA, 527 dehydrodiconiferyl alcohol; HMPPD, 1,2-bis(4-hydroxy-3-methoxyphenyl)-propane-1,3-diol; AV, 528 acetovanillone; FA, ferulate; VN, vanillin; VA, vanillate; SN, syringaldehyde; SA, syringate; PCA, 529 protocatechuate. 

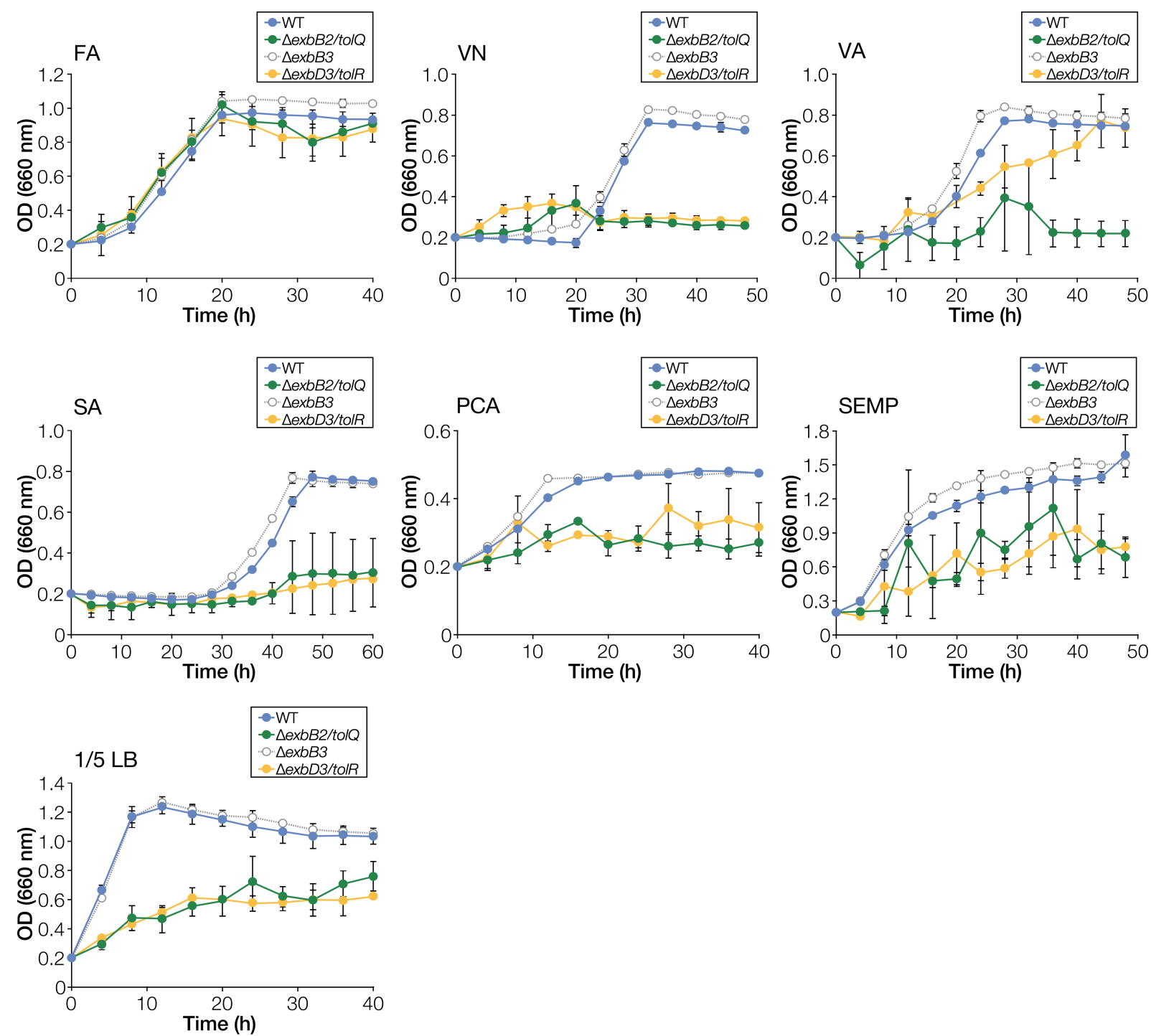

Fig. 2 Growth of $e x b B$ and $e x b D$ mutants on lignin-derived aromatic compounds. Cells of SYK-6, $\triangle e x b B 2 /$ tol $Q, \Delta e x b B 3$, and $\triangle e x b D 3 /$ tolR were cultured in $\mathrm{Wx}$ medium containing $5 \mathrm{mM} F A, \mathrm{VN}, \mathrm{VA}$, SA, or PCA, Wx medium containing SEMP, and diluted LB (1/5 LB). Cell growth was monitored by measuring the $\mathrm{OD}_{660}$. Each value is the average \pm the standard deviation of three independent experiments. 


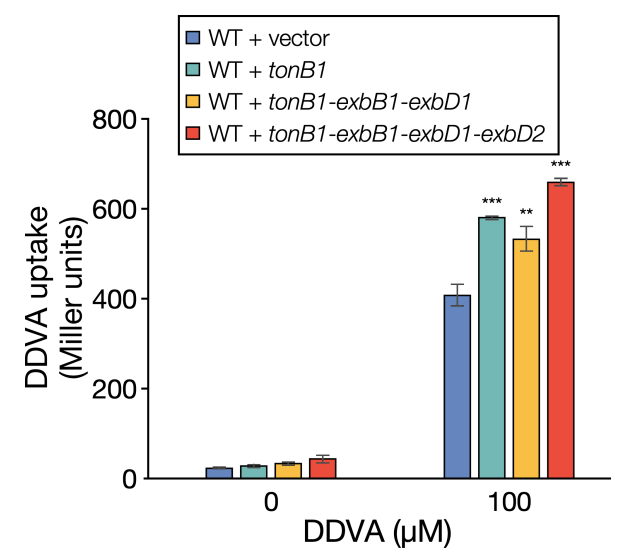

Fig. 3 DDVA uptake by SYK-6 cells overexpressing the tonB1 operon genes. The uptake of DDVA by SYK-6 cells overexpressing the ton $B 1$ operon genes was evaluated using the DDVA sensor plasmid pS-XR, which was constructed by applying the regulatory system of the DDVA catabolic genes $^{24}$. The $\beta$-galactosidase activities of cells of SYK-6(pS-XR + pSEVA338 [vector]), SYK-6(pS-XR + pS-tonB1), SYK-6(pS-XR + pS-t1-D1), and SYK-6(pS-XR + pS-t1-D12) incubated in Wx-SEMP with or without $100 \mu \mathrm{M}$ DDVA were measured. Each value is the average \pm the standard deviation of three independent experiments. Asterisks show statistically significant differences between cells overexpressing the ton $B 1$ operon genes and vector control cells incubated in the presence of $100 \mu \mathrm{M}$ DDVA. ${ }^{* *}, P<0.01,{ }^{* * *}, P<0.001$ (unpaired, two-tailed $t$-test). 

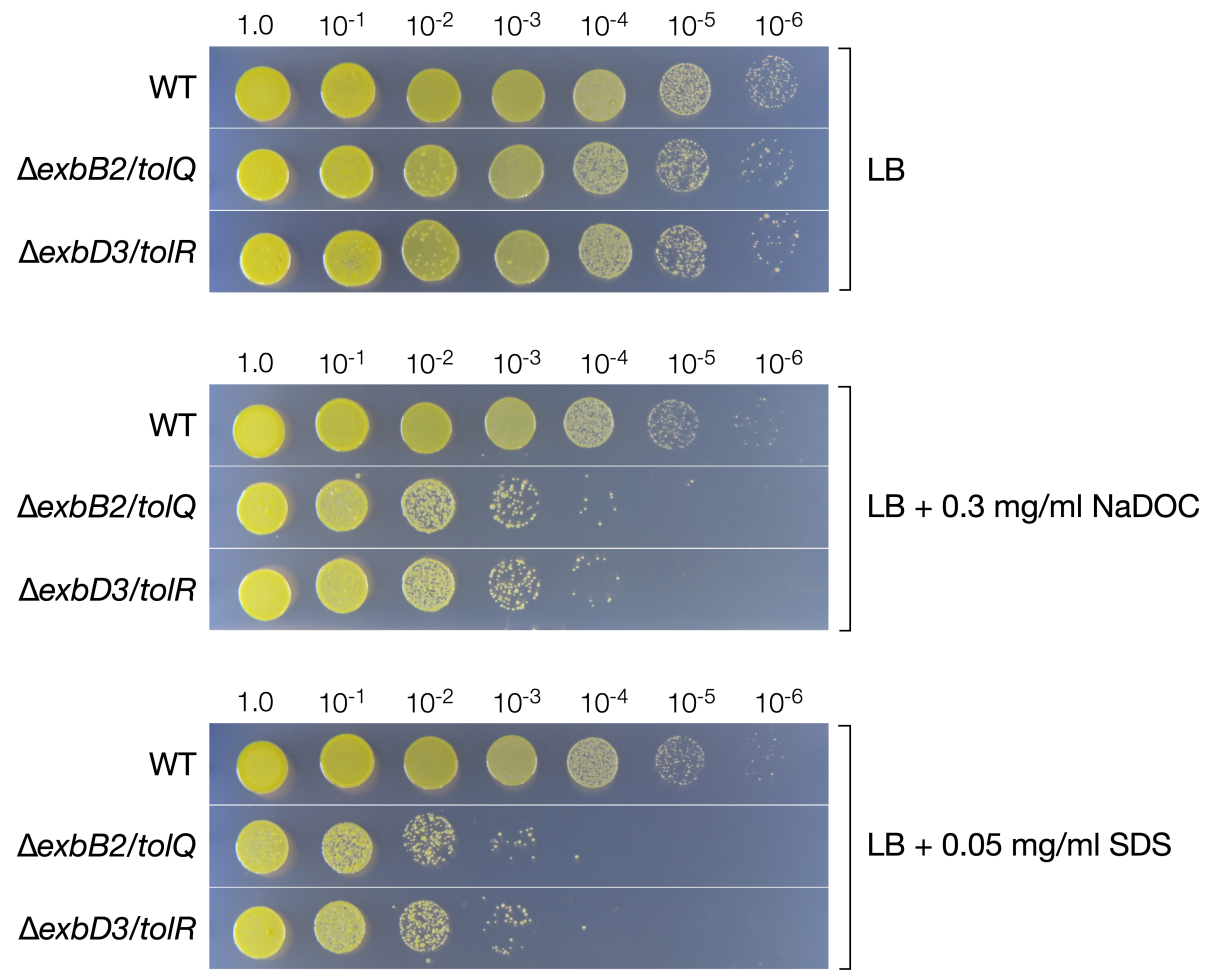

547 Fig. $4 \Delta$ exbB2/tolQ and $\Delta$ exbD3/tolR cells show susceptibility to detergents. The cells of SYK-6, $548 \Delta$ exbB2/tolQ, and $\Delta$ exbD3/tol $R$ were grown in LB with or without $0.3 \mathrm{mg} \mathrm{ml}^{-1}$ of NaDOC or $0.05 \mathrm{mg}$ $549 \mathrm{ml}^{-1}$ SDS. The cell diluted with Wx buffer $(10 \mu \mathrm{l})$ were dropped onto LB agar medium and incubated 550 at $30^{\circ} \mathrm{C}$ for $72 \mathrm{~h}$. 

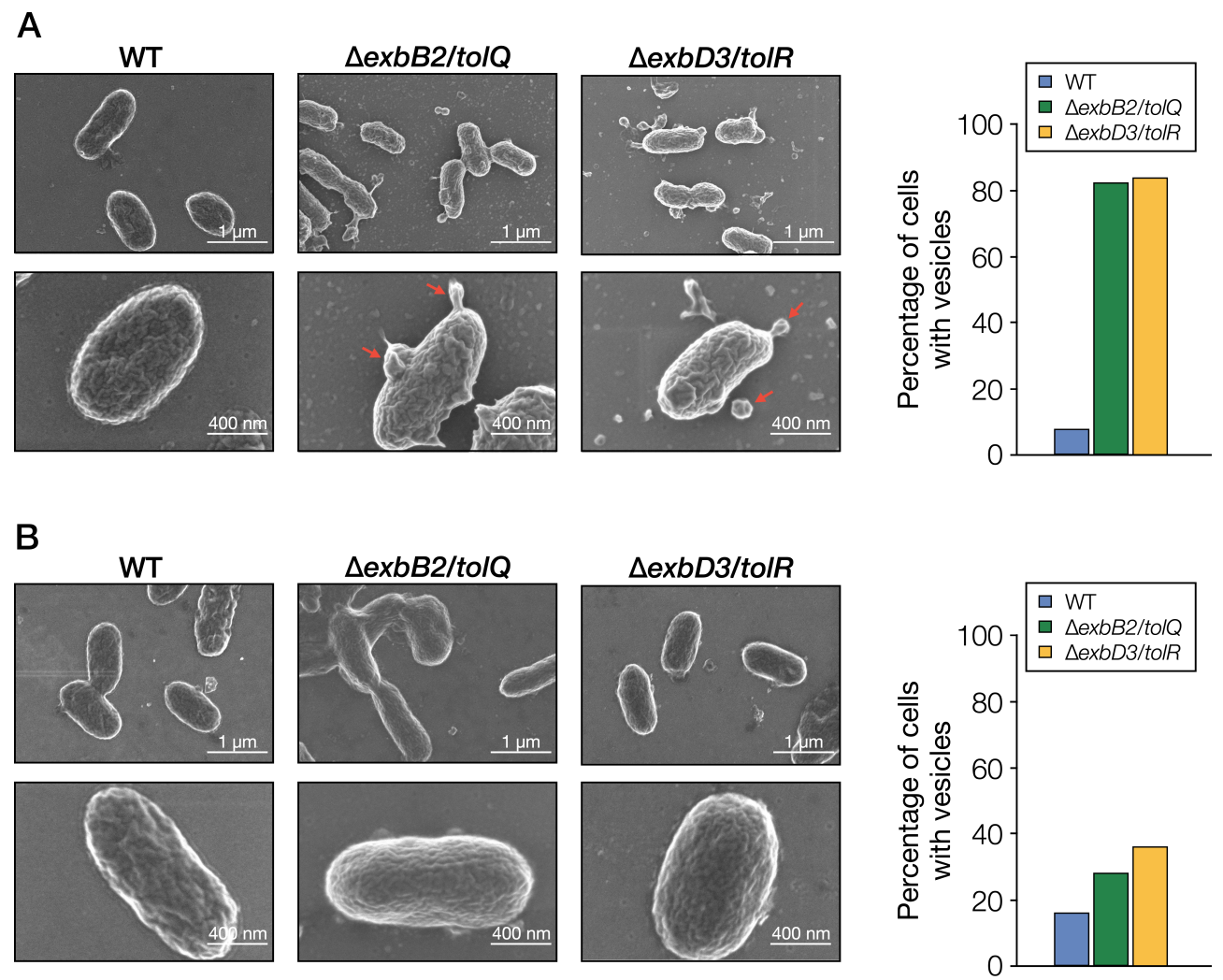

552 Fig. 5 SEM analysis of SYK-6, $\Delta$ exbB2/tolQ, and $\Delta$ exbD3/tolR. (A) FE-SEM images of SYK-6, $553 \Delta$ exbB2/tolQ, and $\Delta$ exbD3/tolR cells grown in LB. Red arrows indicate blebbing vesicles. (B) 554 FE-SEM images of SYK-6, $\Delta e x b B 2 /$ tolQ, and $\Delta e x b D 3 /$ tolR cells grown in LB with $5 \mathrm{mM} F A$. The 555 right figure shows the percentage of cells with vesicles. Outer membrane vesiculation was quantified 556 by imaging 50 bacterial cells and expressed as the percentage of cells with OMV. 


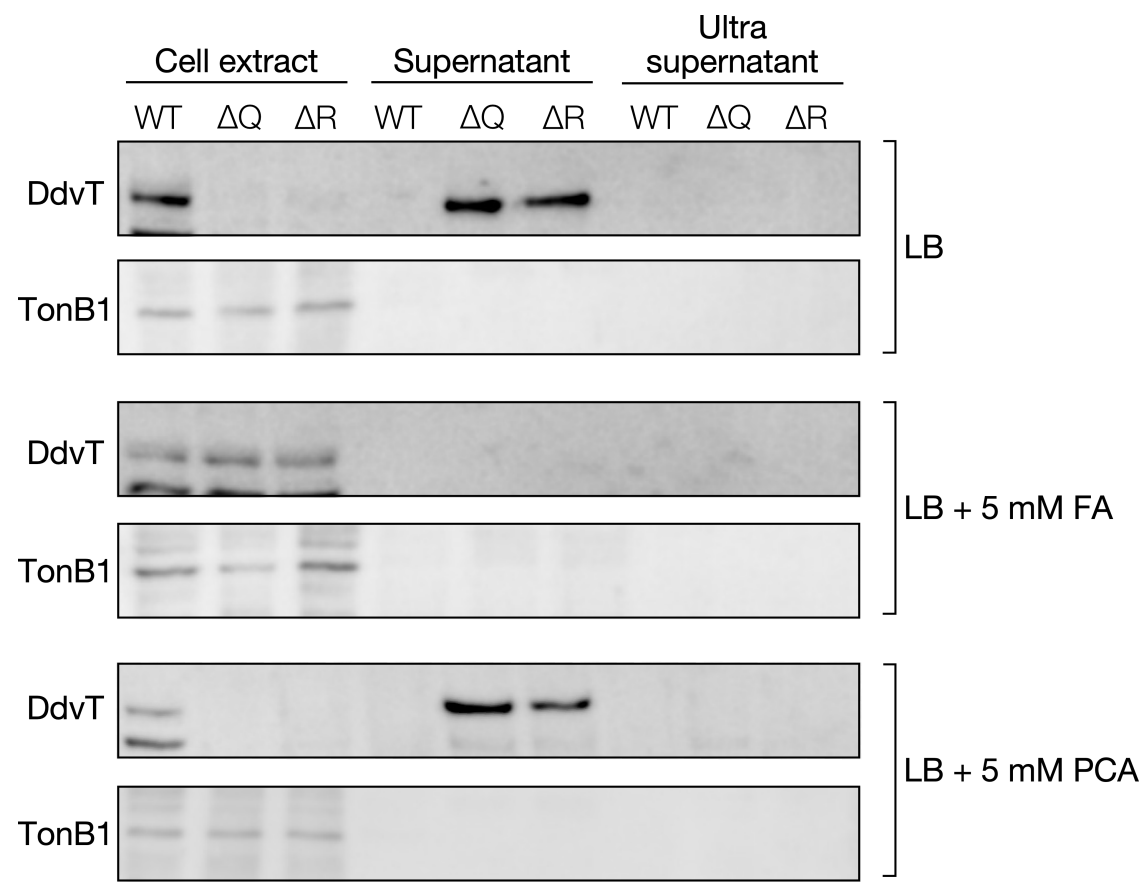

558 Fig. 6 Detection of the outer membrane fraction in the culture supernatants of $\operatorname{exbB} 2 / \mathrm{tol} Q$ and

559 exbD3/tolR mutants. Cells of the wild type (WT), $\Delta \operatorname{exbB2/tolQ~}(\Delta \mathrm{Q})$, and $\Delta \operatorname{exbD3/tolR}(\Delta \mathrm{R})$ were 560 grown in LB with or without $5 \mathrm{mM} \mathrm{FA}$ or PCA. As shown in Fig. S13, after centrifugation, the 561 supernatants of the cultures were collected (supernatant), and the cell extracts were prepared. The 562 supernatants were further ultracentrifuged, and the resulting supernatants were collected 563 (ultrasupernatant). Western blot analysis using anti-DdvT and anti-TonB1 antibodies was performed 564 against cell extracts (10 $\mu \mathrm{g}$ of protein), $20 \mu \mathrm{l}$ of the supernatant, and $20 \mu \mathrm{l}$ of the ultrasupernatant. 


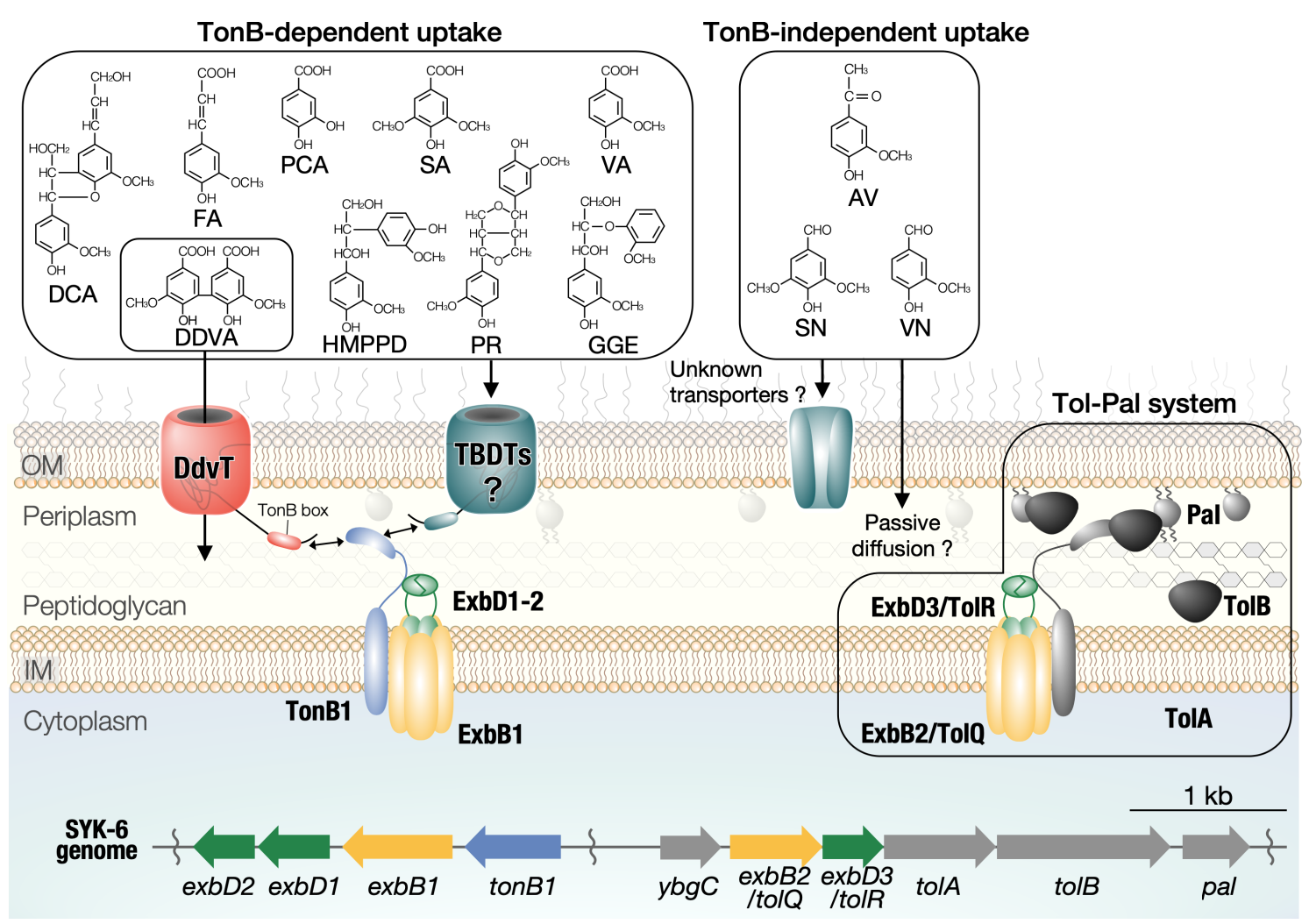

566 Fig. 7 Proposed outer membrane transport of lignin-derived aromatic compounds in SYK-6.

567 Outer membrane transport of GGE, PR, DCA, HMPPD, FA, VA, SA, and PCA is thought to be 568 mediated by TBDTs, and the involvement of TonB1-ExbB1-ExbD1-ExbD2 as the Ton complex is 569 strongly suggested. AV, VN, and SN are presumed to be taken up by transporters other than TBDT or 570 passive diffusion. ExbB2/TolQ and ExbD3/TolR are the components of the Tol-Pal system that stabilizes the outer membrane structure. OM, outer membrane; IM, inner membrane. 